\title{
Prévalence et profils de la multimorbidité au Canada et déterminants associés
}

\author{
K. C. Roberts, M. Sc.; D. P. Rao, M. Sc.; T. L. Bennett, M. Sc.; L. Loukine, M. Sc.; G. C. Jayaraman, Ph. D.
}

Cet article a fait l'objet d'une évaluation par les pairs.

Diffuser cet article sur Twitter

\section{Résumé}

Introduction : La prise en compte de la multimorbidité est de plus en plus reconnue comme un élément fondamental de la prévention et de la prise en charge des affections chroniques. Cette étude porte sur la prévalence et les corrélats de la multimorbidité chez les adultes canadiens en fonction de l'âge et de certains autres déterminants clés.

Méthodologie : Nous avons extrait des données portant sur 105416 adultes canadiens ayant répondu à l'Enquête sur la santé dans les collectivités canadiennes de 2011-2012. Nous les avons analysées en fonction du nombre d'affections concomitantes présentes (deux ou plus ou trois ou plus, sur une liste de neuf) et nous avons cherché à caractériser les déterminants de la multimorbidité à l'aide de régressions.

Résultats : D’après notre analyse, $12,9 \%$ des Canadiens souffraient de deux affections chroniques ou plus et 3,9\% de trois ou plus. Les répondants ayant déclaré souffrir de trois affections chroniques ou plus étaient plus susceptibles d'être des femmes, d'être plus âgés, de faire partie d'un ménage dont le revenu fait partie du quintile le plus faible et dont le niveau de scolarité le plus élevé de l'un des membres était inférieur aux études secondaires. La multimorbidité en lien avec le dénuement social était associée à une cote de 3,7 dans la population en général, mais de 7,5 chez les 35 à 49 ans et de 5,9 chez les 50 à 64 ans, soit la population d'âge moyen.

Analyse : La proportion de Canadiens atteints de multiples affections chroniques étant en augmentation, nous devons adopter, pour étudier les affections chroniques et leurs facteurs en amont, une approche globale qui tienne compte de la multimorbidité, afin d'agir de manière globale et adaptée au contexte pour favoriser une vie saine et une meilleure qualité de vie et pour réduire les coûts des soins de santé et la mortalité. On devrait particulièrement tenir compte du rôle joué par le dénuement social dans l'apparition de la multimorbidité, car non seulement les Canadiens du groupe socioéconomique le plus démuni sont plus susceptibles de souffrir de multimorbidité, mais celle-ci surgira aussi probablement beaucoup plus précocement.

Mots-clés : multimorbidité, Canada, maladie non transmissible, affection chronique

\section{Introduction}

Environ un Canadien sur trois est atteint d'au moins une affection chronique majeure $^{1}$ et on s'attend à ce que cette proportion augmente en raison du vieillissement de la population et de l'augmentation des facteurs de risque liés à ces affections ${ }^{2-4}$. Principale cause de décès à l'échelle de la planète, les affections chroniques étaient à l'origine de 38 millions (68\%) de décès en 2012 et L'Organisation mondiale de la santé prévoit que le nombre annuel total de décès

\section{Principales constatations}

- En 2011-2012, 12,9 \% des adultes canadiens de 20 ans ou plus souffraient de deux affections chroniques ou plus, et 3,9\% de trois ou plus.

- L'arthrite, les troubles de l'humeur ou l'anxiété ainsi que l'asthme étaient les affections chroniques les plus fréquentes, et elles étaient également dominantes dans les associations de multimorbidité.

- La prévalence de la multimorbidité augmentait avec l'âge des répondants, avec la faiblesse du revenu de leur ménage et avec celle du plus haut niveau de scolarité au sein de leur ménage.

- La multimorbidité devenant la norme chez les personnes atteintes d'affections chroniques, utiliser une approche globale portant sur l'ensemble de ces affections chroniques plutôt que sur chacune séparément s'avère de plus en plus pertinent.

causés par une affection chronique atteindra les 52 millions d'ici $2030^{5}$. Au Canada, les cardiopathies, les accidents vasculaires cérébraux (AVC), le cancer, les affections respiratoires chroniques et le diabète étaient à l'origine de $62,7 \%$ des décès en $2011^{6}$. La multimorbidité - soit la présence simultanée de deux affections chroniques ou plus - complexifie la prévention et la prise en charge, en partie parce que les personnes atteintes de plusieurs affections risquent de souffrir de davantage d'effets indésirables sur la santé et d'avoir plus souvent besoin d'être hospitalisées et de recevoir des soins de santé ${ }^{7}$. 
Quoique la multimorbidité soit de plus en plus considérée comme un enjeu clé en matière de santé publique, son épidémiologie n'est pas bien connue. Les estimations de la prévalence de la multimorbidité sont très variables (entre $13,1 \%{ }^{8}$ et $90 \%{ }^{9}$ ), essentiellement en raison des différences dans les définitions de cas, les sources de données et les populations étudiées ${ }^{10}$. Comme on mesure de manière générale la multimorbidité en demandant à une personne si elle est atteinte de deux affections chroniques ou plus figurant sur une liste prédéfinie $^{6}$, le choix des affections inscrites sur la liste peut influencer les estimations de prévalence. Ainsi, $36 \%$ des Albertains seraient atteints de deux affections chroniques ou plus d'après Agborsangaya et ses collaborateurs $^{11}$, mais leur définition inclut l'obésité, dont la prévalence est à elle seule de $28,1 \%$. Restreindre l'échantillon à certaines sous-populations, par exemple les personnes vivant en établissement ou les aînés, influencerait également les estimations $^{9,12-16}$.

L'objectif principal de notre étude était d'examiner la prévalence de la multimorbidité dans la population canadienne adulte en fonction de plusieurs variables sociodémographiques et de plusieurs comportements à risque. l'objectif secondaire était d'examiner la prévalence de paires et de triades d'affections chroniques.

\section{Méthodologie}

\section{Source de données}

Notre étude, fondée sur une analyse de la population canadienne de 20 ans et plus, fait appel aux données de l'Enquête sur la santé dans les collectivités canadiennes (ESCC) de 2011-2012. l'ESCC est une enquête populationnelle transversale sur la santé réalisée auprès des Canadiens de 12 ans et plus et excluant les personnes vivant en résidence ou en établissement de soins de longue durée ainsi que celles vivant sur une réserve, les militaires à temps plein des Forces armées canadiennes et les résidents civils des bases militaires. L'enquête a été conçue pour permettre des estimations à l'échelle nationale, à l'échelle provinciale et à l'échelle des 110 régions sanitaires du Canada. La méthodologie de l'étude est décrite en détail dans un autre article ${ }^{17}$. Notre stratégie de répartition de l'échantillon à plusieurs degrés assure une pondération relativement égale aux différentes régions sanitaires et aux provinces.

\section{Variables à l'étude}

En vue de déterminer la prévalence de certaines affections chroniques, on a interrogé les répondants de l'ESCC sur la présence d'une affection affectant leur état de santé à long terme, c'est-à-dire « durant, ou susceptible de durer, six mois ou plus et diagnostiquée par un professionnel de la santé ». On les a également questionnés sur leurs caractéristiques individuelles et socioéconomiques, notamment leur sexe et leur âge, les caractéristiques de leur ménage (c.-à-d. le nombre de personnes composant leur ménage, le plus haut niveau de scolarité atteint par un membre de leur ménage et le revenu de leur ménage) et leur identité autochtone (ou non autochtone). Pour notre analyse, nous avons attribué une valeur relative au statut socioéconomique de chaque répondant en fonction du revenu total et des caractéristiques de son ménage (en utilisant les quintiles du revenu de Statistique Canada) afin de comparer les revenus les plus bas et les revenus les plus élevés. Les données de l'Enquête que nous avons utilisées sont autodéclarées, aussi bien en ce qui concerne les comportements, à savoir le tabagisme (fumeur quotidien ou occasionnel / non-fumeur), les habitudes alimentaires ( 5 portions ou plus de fruits et de légumes par jour ${ }^{18} /$ moins de 5), l'activité physique pendant les loisirs (actif ou modérément actif / inactif) et l'obésité (indice de masse corporelle supérieur ou égal à 30), qu'en ce qui concerne les états de santé à risque, à savoir l'hypertension et le stress (pas du tout, pas tellement ou un peu stressé/ beaucoup ou extrêmement stressé $)^{18}$. Le choix des facteurs de risque comportementaux liés aux maladies chroniques s'est effectué dans le cadre d'un processus de consultation entre l'Agence de la santé publique du Canada et divers intervenants pertinents ${ }^{19}$.

\section{Définition de cas de la multimorbidité}

Nous avons suivi la définition de la multimorbidité établie en 2012 par un groupe de travail réunissant divers experts et coordonné par l'Agence de la santé publique du Canada, à savoir la coexistence de deux affections chroniques ou plus ou de trois affections chroniques ou plus parmi neuf affections présélectionnées pour leur chronicité, leur prévalence élevée au Canada, l'importance de leurs conséquences sociales ou économiques ou leur réceptivité à la prévention primaire ${ }^{20}$. En suivant ces critères et en tenant compte de la disponibilité des données dans l'ESCC, nous avons choisi les neuf affections suivantes: l'arthrite, un trouble mental (trouble de l'humeur ou anxiété), l'asthme, le diabète sucré, une cardiopathie, une maladie pulmonaire obstructive chronique (MPOC, données accessibles seulement pour les sujets de 35 ans et plus), un cancer, un AVC et la maladie d'Alzheimer ou une démence associée. Nous avons analysé les données en utilisant la définition de cas correspondant à deux affections chroniques ou plus et celle correspondant à trois affections chroniques ou plus.

\section{Analyses statistiques}

La prévalence de la multimorbidité a été estimée à la fois pour l'ensemble de la population et par sexe. Les paires et les triades les plus fréquentes dans chaque groupe d'âge (20 à 34 ans, 35 à 49 ans, 50 à 64 ans, 65 ans et plus) $)^{21}$ ont été repérées. Nous présentons ici les cinq combinaisons principales touchant au moins $5 \%$ des Canadiens atteints de multimorbidité au sein d'une même association (paire ou triade) et d'un même groupe d'âge.

On a eu recours à des modèles logistiques univariés pour examiner la relation entre multimorbidité et divers facteurs de risque sociodémographiques, sanitaires et comportementaux : l'âge, le sexe, le revenu du ménage, le niveau de scolarité le plus élevé au sein du ménage, l'appartenance ou non à un groupe autochtone, le milieu de résidence (urbain ou rural), l'activité physique, la consommation de fruits et de légumes, le tabagisme, le degré de stress, l'hypertension et l'obésité. Les rapports de cotes ont été utilisés pour mesurer les associations et le test du chi carré de Wald pour effectuer les analyses 
statistiques. Nous avons réussi à repérer les facteurs associés de façon significative $(p<0,05)$ à la multimorbidité (définie par le seuil conservateur de trois affections ou plus plutôt que deux, une ou aucune) grâce à un modèle multivarié fondé sur une régression logistique descendante pas à pas. Les analyses multivariées ont été stratifiées selon l'âge afin de tenir compte des modifications qui lui sont potentiellement liées. On a utilisé le test du chi carré pour examiner la tendance relative à l'activité physique par groupe d'âge.

Les données de l'ESCC ont été pondérées afin d'être représentatives de la population canadienne, et des intervalles de confiance (IC) à $95 \%$ des estimations de la prévalence et des rapports de cotes (RC) ont été calculés pour chaque analyse à l'aide d'une méthode de rééchantillonnage bootstrap (à 500 répliques) pour tenir compte de la complexité de la conception de l'enquête. Les analyses statistiques ont été effectuées à l'aide des énoncés de procédure SAS (SAS EG, version 5.1, SAS Institute Inc., Cary, Caroline du Nord, É-U).

\section{Résultats}

\section{Prévalence et distribution de la multimorbidité en fonction des variables sociodémographiques et des facteurs de risque}

En 2011-2012, 12,9 \% (IC à 95 \% : 12,6 à 13,2; données non présentées) des Canadiens de 20 ans et plus ( $\mathrm{n}=105416$ ) ont déclaré souffrir de deux affections chroniques ou plus et 3,9\% (IC à $95 \%: 3,7$ à 4,1 ; données non présentées) de trois ou plus. Quelle qu'ait été la définition de cas employée (deux affections ou plus ou trois affections ou plus), la prévalence de la multimorbidité suivait des tendances similaires pour toutes les caractéristiques sociodémographiques et tous les facteurs de risque (tableau 1). Parmi les répondants ayant fait état de trois affections ou plus, la prévalence de la multimorbidité était significativement plus élevée $(p<0,05)$ chez les femmes $(4,5 \%$ contre $3,3 \%$ chez les hommes; $\mathrm{RC}=1,3$; IC à $95 \%$ : 1,2 à 1,4), chez les plus âgés $(11,3 \%$ chez les 65 ans et plus contre $0,4 \%$ chez les 20 à 34 ans; $\mathrm{RC}=35,0$; IC à $95 \%$ : 26,2 à 46,8), pour le quintile de revenu le plus faible $(7,4 \%$ contre $1,5 \%$ pour le quintile de revenu le plus élevé; RC = 3,7; IC à $95 \%: 3,1$ à 4,5), chez ceux relevant d'un ménage où le niveau de scolarité le plus élevé était inférieur au diplôme d'études secondaires (11,6 \% contre 2,9\% chez ceux relevant d'un ménage où le niveau de scolarité était celui d'études postsecondaires; $\quad \mathrm{RC}=1,8 ; \quad$ IC à $95 \%$ : 1,6 à 2,1), chez ceux s'étant déclarés autochtones $(6,6 \%$ contre $4,0 \%$ chez les non-Autochtones; $\quad \mathrm{RC}=2,7 ; \quad \mathrm{IC}$ à $95 \%$ : $2,2$ à 3,4$)$ et chez ceux nés au Canada (4,2\% contre $3,4 \%$ chez les immigrants vivant au Canada depuis 5 ans ou plus; $\mathrm{RC}=0,7$; IC à $95 \%: 0,6$ à 0,8).

L'hypertension $(\mathrm{RC}=3,2$; IC à $95 \%: 2,9$ à $3,5)$, l'obésité $(\mathrm{RC}=2,7$; IC à $95 \%: 2,4$ à $3,0)$, l'inactivité physique $(\mathrm{RC}=1,7$; IC à $95 \%$ : 1,6 à 1,9), un niveau de stressé élevé (RC = 2,5; IC à $95 \%: 2,2$ à 2,8), la consommation de moins de cinq portions de fruits et de légumes par jour ( $\mathrm{RC}=1,2$; IC à $95 \%: 1,1$ à 1,4$)$ et le tabagisme $(\mathrm{RC}=2,1$; IC à $95 \%: 1,9$ à 2,4) se sont révélés significativement associés à la multimorbidité lorsque nous avons utilisé la définition de cas de trois affections ou plus (tableau 1).

Parmi les adultes de 20 à 65 ans, 2,2\% (IC à $95 \%: 2,1$ à 2,4) ont déclaré souffrir de trois affections chroniques ou plus et 8,8 \% (IC à $95 \%$ : 8,4 à 9,1) de deux ou plus (données non présentées).

\section{Prévalence des affections chroniques et associations les plus fréquentes}

Nous avons estimé la prévalence de certaines affections chroniques pour déterminer la multimorbidité pour l'ensemble de la population. L'arthrite $(17,6 \%$; IC à $95 \%$ : 17,2 à 17,9), les troubles de l'humeur ou l'anxiété $(11,2 \%$; IC à $95 \%$ : $10,8$ à 11,5$)$ et l'asthme $(8,1 \%$; IC à $95 \%$ : 7,8 à 8,4) étaient les plus fréquentes. Chez les femmes étaient significativement plus élevées les fréquences de l'asthme (9,4 \% contre $6,7 \%$ chez les hommes), de l'arthrite $(21,7 \%$ contre $13,3 \%)$, de la MPOC $(3,4 \%$ contre $2,7 \%)$ et des troubles de l'humeur ou l'anxiété (14,1\% contre $8,1 \%$ ), alors que chez les hommes étaient plus élevées les fréquences du diabète sucré $(7,8 \%$ contre $6,0 \%$ chez les femmes) et des cardiopathies (6,4\% contre 4,6\%) (tableau 2).

Les associations d'affections chroniques les plus fréquemment observées au sein de la population étaient variables selon les groupes d'âge (tableau 3). La paire d'affections la plus courante était l'arthrite et les troubles de l'humeur ou l'anxiété (37,8 \%; IC à $95 \%$ : 35,6 à 40,0), suivie des combinaisons arthrite et cardiopathie $(35,2 \%$; IC à $95 \%: 33,2$ à 37,2) et arthrite et diabète $(35,2 \%$; IC à $95 \%$ : 33,1 à 37,3). La triade la plus fréquemment mentionnée était arthrite, asthme et troubles de l'humeur ou anxiété (15,5\%; IC à $95 \%$ : 13,8 à 17,1), suivie des combinaisons arthrite, diabète et cardiopathie (15,1\%; IC à $95 \%$ : 13,6 à 16,7) et asthme, arthrite et MPOC $(14,7 \%$; IC à $95 \%: 13,2$ à 16,2).

Chez les jeunes Canadiens (20 à 34 ans), les troubles de l'humeur ou l'anxiété représentaient 51,6\% (IC à $95 \%$ : 49,2 à 53,9; données disponibles sur demande) des affections multiples déclarées. Chez les répondants âgés de 35 ans et plus, les combinaisons d'affections étaient plus hétérogènes et la prévalence de combinaisons précises d'affections diminuait avec l'âge. La contribution des troubles de l'humeur ou de l'anxiété au fardeau total de la multimorbidité déclinait jusqu'à $25,3 \%$ (IC à $95 \%$ : 24,5 à 26,1; données disponibles sur demande) chez les plus âgés. Plus une affection donnée était fréquente ou prévalente au sein de la population (voir le tableau 2), plus elle était susceptible de contribuer à la multimorbidité - ce qui n'est guère surprenant. Ceci explique que la contribution d'affections comme le diabète ou la MPOC au fardeau de la multimorbidité était plus importante chez les groupes plus âgés. Il est important de noter que les cinq combinaisons principales d'affections suivaient la même tendance générale chez les deux sexes, sauf pour la cinquième combinaison, où l'on retrouve diabète et cardiopathie chez les hommes mais arthrite et MPOC chez les femmes (données non présentées).

\section{Déterminants de la multimorbidité (trois affections chroniques ou plus)}

Après ajustement du modèle pour l'ensemble des variables, nous avons 
TABLEAU 1

Distribution et prévalence de la multimorbiditéa en fonction des caractéristiques sociodémographiques (ESCC 2011-2012, population de 20 ans et plus)

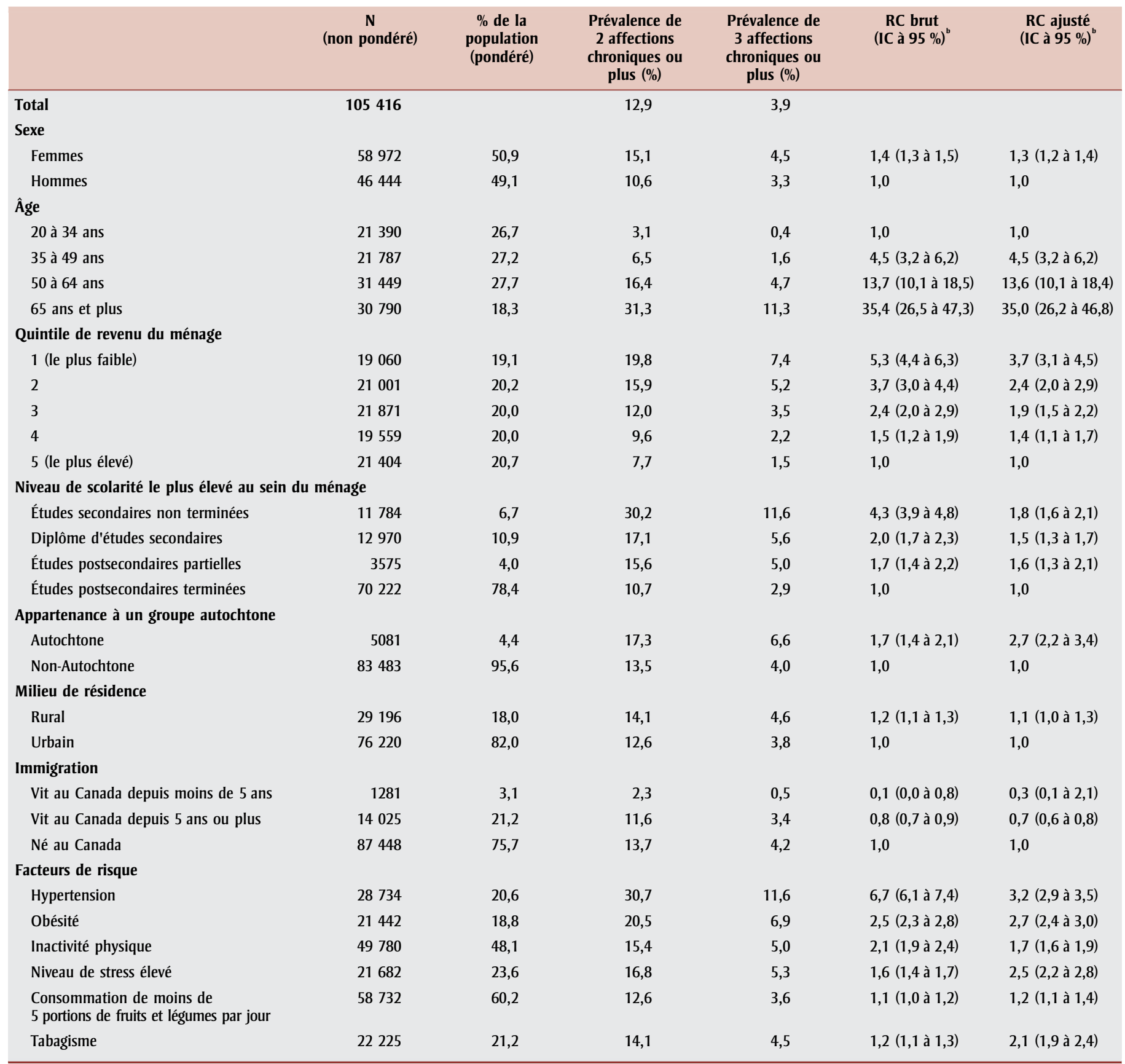

Abréviations : AVC, accident vasculaire cérébral; ESCC, Enquête sur la santé dans les collectivités canadiennes; IC, intervalle de confiance; RC, rapport de cotes.

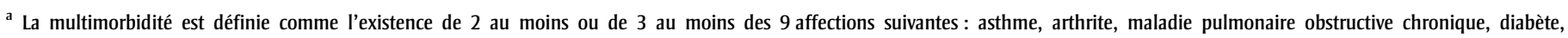
cardiopathie, trouble mental (trouble de l'humeur ou anxiété), maladie d'Alzheimer ou démences associées, cancer et AVC.

b Pour la prévalence de 3 affections chroniques ou plus, ajustement pour l'âge et le sexe.

constaté une association statistiquement significative entre la multimorbidité et le sexe, l'âge, le niveau de revenu du ménage, le fait d'être autochtone, l'activité physique, le tabagisme, le niveau de stress et l'obésité. L'analyse de la stratification selon l'âge laisse penser que l'inactivité physique contribue progressivement - et de manière significative à partir de 50 ans - à l'augmentation de la prévalence de la multimorbidité (tableau $4 ; p<0,001$ ). 
TABLEAU 2

Prévalence des affections chroniques dans l'ensemble de la population (20 ans et plus) et chez les répondants atteints de multimorbidité ( 2 affections chroniques ou plus ou 3 affections chroniques ou plus) (ESCC 2011-2012)

\begin{tabular}{|c|c|c|c|c|c|c|}
\hline \multirow[t]{2}{*}{ Affection chronique } & \multicolumn{2}{|c|}{ Ensemble de la population } & \multicolumn{2}{|c|}{ Hommes } & \multicolumn{2}{|c|}{ Femmes } \\
\hline & $\mathbf{N}$ & Prévalence (\%) & $\mathbf{N}$ & Prévalence (\%) & $\mathbf{N}$ & Prévalence (\%) \\
\hline Arthrite & 25880 & $17,6(17,2$ à 17,9$)$ & 8858 & $13,3(12,8$ à 13,7$)$ & 17022 & $21,7(21,1$ à 22,3$)$ \\
\hline Trouble mental & 12652 & $11,2(10,8$ à 11,5$)$ & 4147 & $8,1(7,6$ à 8,5$)$ & 8505 & $14,1(13,6$ à 14,6$)$ \\
\hline Asthme & 9000 & $8,1(7,8$ à 8,4$)$ & 3163 & $6,7(6,3$ à 7,1$)$ & 5837 & $9,4(9,0$ à 9,9$)$ \\
\hline Diabète sucré & 9450 & $6,9(6,6$ à 7,1$)$ & 4828 & $7,8(7,4$ à 8,1$)$ & 4622 & 6,0 (5,7 à 6,4) \\
\hline Cardiopathie & 8218 & $5,5(5,3$ à 5,7$)$ & 4289 & $6,4(6,0$ à 6,8$)$ & 3929 & $4,6(4,3$ à 4,9) \\
\hline MPOC & 4701 & $3,1(2,9$ à 3,2) & 1859 & $2,7(2,5$ à 2,9$)$ & 2842 & $3,4(3,2$ à 3,7$)$ \\
\hline Cancer & 3694 & $2,5(0,4$ à 2,7$)$ & 1476 & $2,2(2,0$ à 2,4$)$ & 2218 & $2,9(2,6$ à 3,1$)$ \\
\hline AVC & 1944 & $1,3(1,2$ à 1,4$)$ & 925 & $1,3(1,2$ à 1,4$)$ & 1019 & $1,3(1,1$ à 1,5$)$ \\
\hline Maladie d'Alzheimer et démences associées & 634 & $0,5(0,4$ à 0,6$)$ & 330 & $0,5(0,4$ à 0,7$)$ & 304 & $0,5(0,4$ à 0,6$)$ \\
\hline
\end{tabular}

Abréviations : AVC, accident vasculaire cérébral; ESCC, Enquête sur la santé dans les collectivités canadiennes; MPOC, maladie pulmonaire obstructive chronique.

Remarque : Les troubles mentaux comprennent les troubles de l'humeur et l'anxiété.

TABLEAU 3

Prévalence des paires et des triades d'affections chroniques les plus fréquentes, selon les groupes d'âge (ESCC 2011-2012, population de 20 ans et plus)

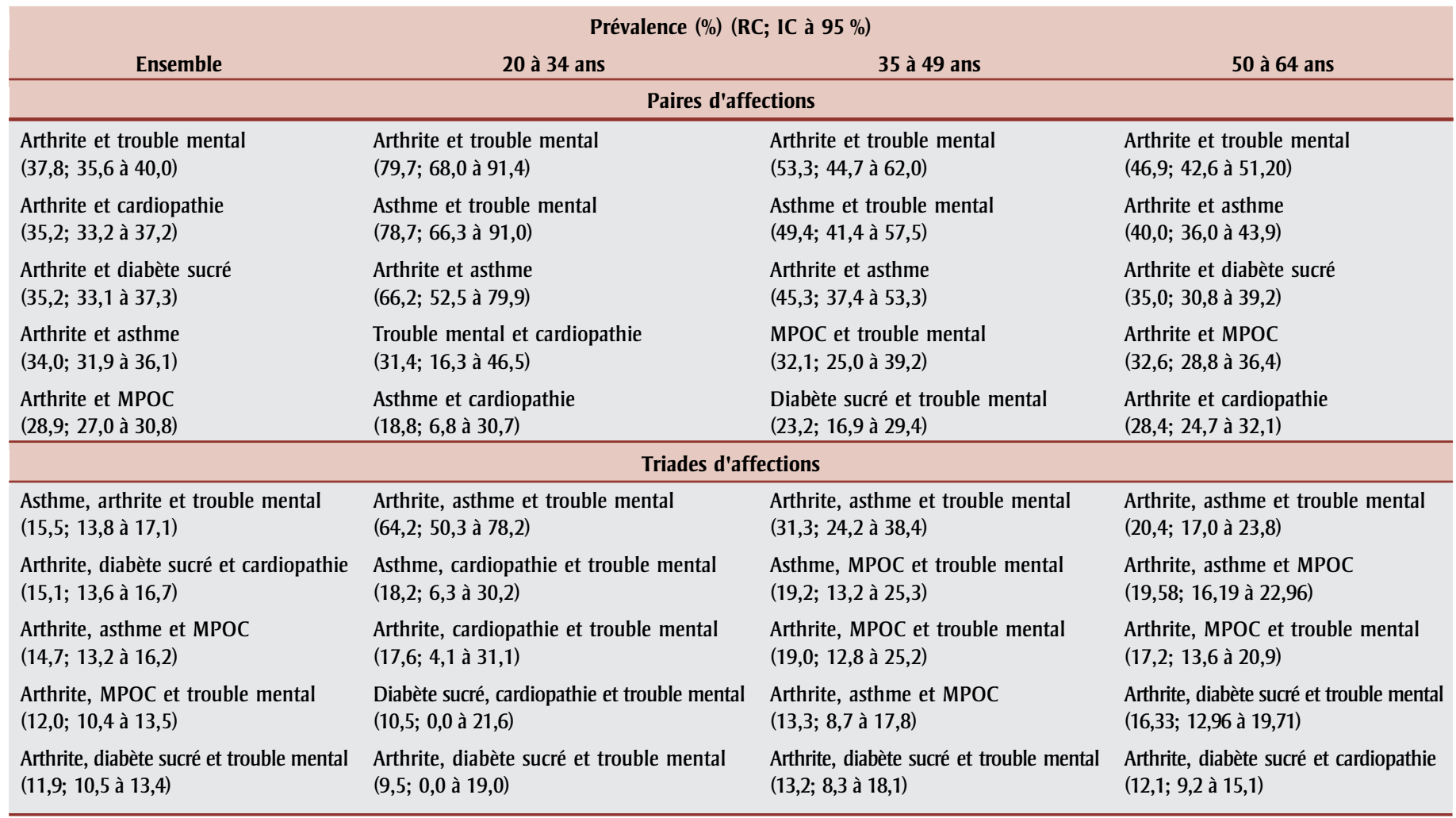

Abréviations : ESCC, Enquête sur la santé dans les collectivités canadiennes; IC, intervalle de confiance; MPOC, maladie pulmonaire obstructive chronique; RC, rapport de cotes.

Remarques : Les troubles mentaux comprennent les troubles de l'humeur et l'anxiété.

Seules les combinaisons d'affections ayant une prévalence supérieure à $5 \%$ pour chaque groupe d'âge sont incluses dans le tableau.

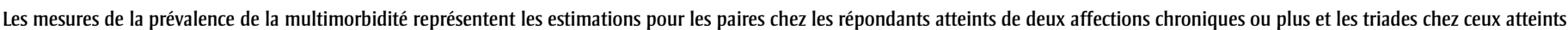
de trois ou plus. 
TABLEAU 4

Analyse de l'association entre les caractéristiques sociodémographiques, les facteurs de risque comportementaux et la multimorbidité (trois affections chroniques ou plus) selon l'âge (ESCC 2011-2012)

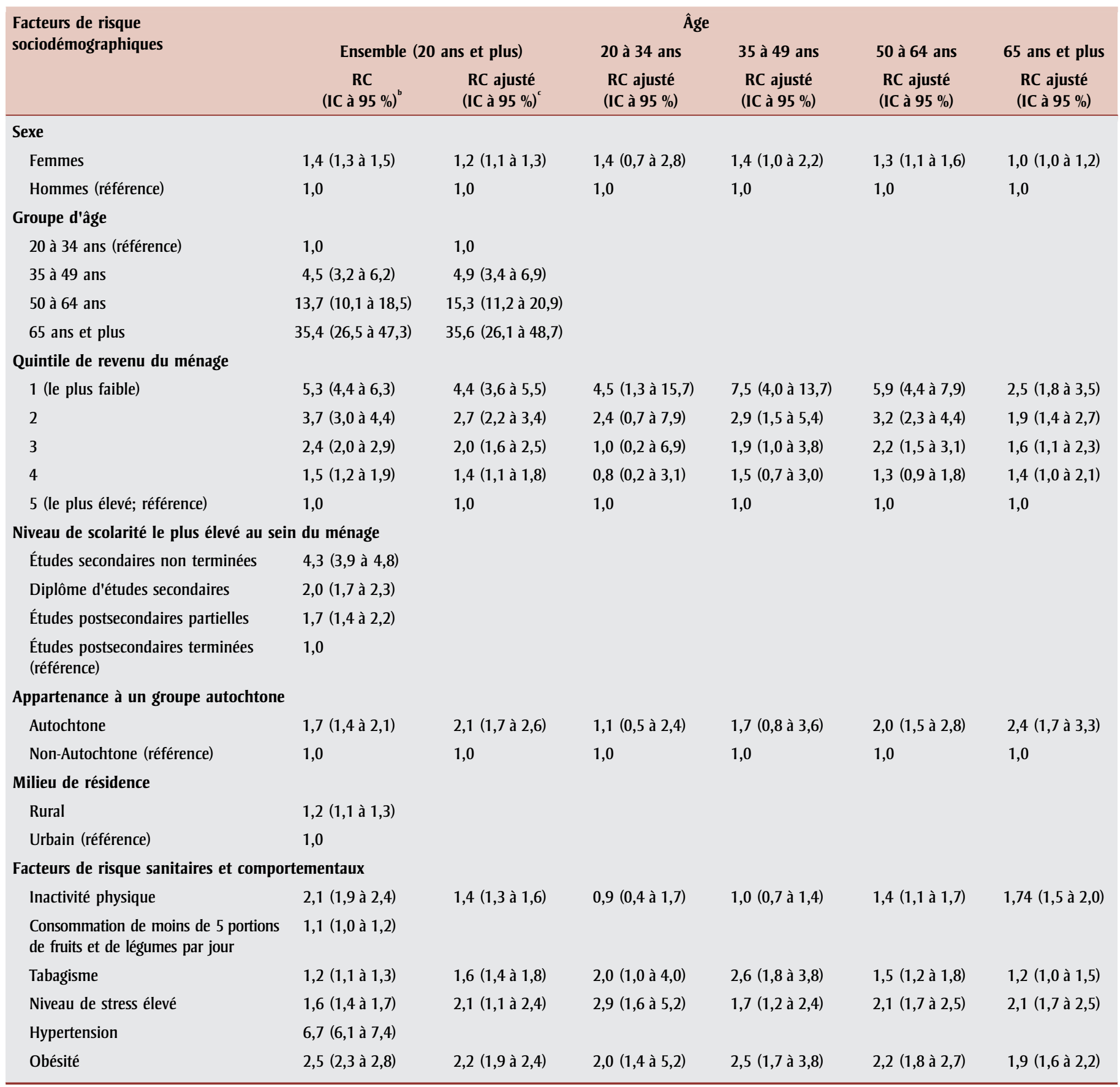

Abréviations : AVC, accident vasculaire cérébral; ESCC, Enquête sur la santé dans les collectivités canadiennes; IC, intervalle de confiance; MPOC, maladie pulmonaire obstructive chronique; $\mathrm{RC}$, rapport de cotes.

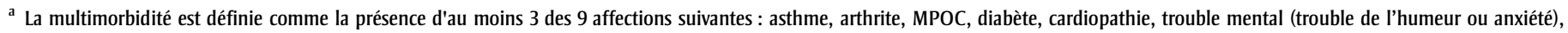
maladie d'Alzheimer ou démences associées, cancer, AVC.

b Rapport de cotes brut.

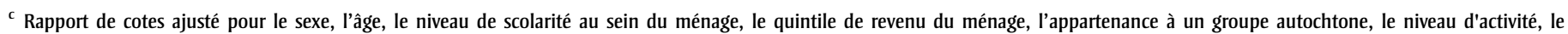
tabagisme, le stress, l'hypertension et l'obésité.

Chez les plus jeunes (20 à 34 ans), un quintile de faible revenu était le seul facteur de risque individuel significativement associé à la multimorbidité
( $R C$ ajusté pour le plus bas quintile $=4,5$; IC à $95 \%$ : 1,3 à 15,7). Des problèmes de santé comme l'obésité (RC ajusté $=2,7$; IC à $95 \%: 1,4$ à 5,2) et un niveau de stress élevé (RC ajusté $=2,9 ; \quad$ IC à $95 \%: 1,6$ à 5,2) étaient également significativement associés à la multimorbidité. 
Chez les 35 à 49 ans, un faible revenu était également un facteur contribuant de manière significative à la multimorbidité (RC ajusté pour le plus bas quintile $=7,5$; IC à $95 \%: 4,0$ à 13,7), tout comme l'obésité (RC ajusté $=2,5$; IC à $95 \%: 1,7$ à 3,8), un niveau de stress élevé ( $\mathrm{RC}$ ajusté $=1,7$; IC à $95 \%$ : 1,2 à 2,4) et le tabagisme $(\mathrm{RC}$ ajusté $=2,6$; IC à $95 \%: 1,8$ à 3,8).

Chez les 50 à 64 ans, le fait d'être une femme $\quad($ RC ajusté $=1,3$; $\quad$ IC à $95 \%$ : 1,1 à 1,6), un faible revenu (RC ajusté pour le plus faible quintile de revenu $=5,3$, IC à $95 \%$ : 4,4 à 7,9) et le fait d'être autochtone (RC ajusté $=2,0$, IC à $95 \%$ : 1,5 à 2,8) étaient significativement associés à la multimorbidité (tableau 4). L'inactivité physique est apparue comme un facteur de risque significatif (RC ajusté $=1,4 ; \quad$ IC à $95 \%: 1,1$ à 1,7$)$. Le tabagisme (RC ajusté $=1,5$; IC à $95 \%$ : $1,2$ à 1,8$)$, un niveau de stress élevé ( $\mathrm{RC}$ ajusté $=2,1$; IC à $95 \%: 1,7$ à 2,5) et l'obésité (RC ajusté = 2,24; IC à $95 \%$ : 1,8 à 2,7 ) sont demeurés significativement associés à la multimorbidité.

Chez les 65 ans et plus, un faible revenu (RC ajusté pour le plus faible quintile $=2,5$; IC à $95 \%: 1,8$ à 3,5) et le fait d'être autochtone (RC ajusté $=2,4$; IC à $95 \%: 1,7$ à 3,3 ) étaient significativement associés à la multimorbidité. C'était également le cas de facteurs de risque comme l'inactivité physique ( $\mathrm{RC}$ ajusté $=1,7$; IC à $95 \%: 1,5$ à 2,0), un niveau de stress élevé (RC ajusté $=2,1$, IC à $95 \%$ : 1,7 à 2,5) et l'obésité (RC ajusté $=1,9$; IC à $95 \%: 1,6$ à 2,2).

\section{Analyse}

Les analyses présentées dans cet article contribuent à justifier le fait que l'on cherche à améliorer la mesure de la multimorbidité pour répondre aux besoins en matière de planification et de politiques $^{22}$. Notons que la multimorbidité implique la coexistence de multiples affections sans que l'une soit plus importante que les autres, contrairement à la définition de la comorbidité, fondée sur le choix d'une affection principale et d'une ou plusieurs affections secondaires. Les estimations de la prévalence concernant la multimorbidité sont très variables dans la littérature ${ }^{10}$, probablement, nous l'avons $\mathrm{vu}$, en raison de différences méthodologiques, notamment les caractéristiques de la population, la taille de l'échantillon et les méthodes de recrutement, ainsi qu'en raison des définitions de la multimorbidité qui ont été appliquées ${ }^{10}$. D’après notre analyse de la multimorbidité, fondée sur des données autodéclarées représentatives de la population canadienne adulte, en 2011-2012, 12,9 \% des répondants (IC à $95 \%$ : 12,6 à 13,2) souffraient de deux affections chroniques ou plus et 3,9\% (IC à $95 \%: 3,7$ à 4,1) de trois affections ou plus. Si la forte association entre l'âge et la multimorbidité est déjà bien connue ${ }^{12-14}$, certaines constatations clés de notre étude méritent un examen plus attentif.

En premier lieu, même si la multimorbidité s'est révélée plus importante chez les répondants les plus âgés ( 65 ans et plus), $2,2 \%$ des Canadiens de 20 à 65 ans ont déclaré souffrir de trois affections chroniques ou plus et $8,8 \%$ de deux affections chroniques ou plus, ce qui concorde avec les résultats d'études canadiennes antérieures $^{11}$.

En deuxième lieu, l'association entre certains facteurs de risque et la multimorbidité augmente avec l'âge, même si plusieurs caractéristiques individuelles (comme un faible revenu) se sont révélées significativement associées à la multimorbidité quel que soit l'âge. Ainsi, dans la population prise dans son ensemble, la multimorbidité due au dénuement social était associée à une cote de 3,7, mais cette cote était nettement plus élevée chez les personnes d'âge moyen (7,5 chez les 35 à 49 ans et 5,9 chez les 50 à 64 ans). Cette différenciation de prévalence de la multimorbidité à l'âge moyen associée au dénuement social fait écho aux résultats de Barnett et de ses collaborateurs ${ }^{23}$, selon lesquels l'apparition de la multimorbidité survient de 10 à 15 ans plus tôt chez les personnes vivant dans les conditions socioéconomiques les plus difficiles. Chez elles, les besoins en matière de prise en charge clinique sont susceptibles d'être plus importants et de se manifester plus tôt, et la multimorbidité est susceptible d'être de plus longue durée. Nos analyses ne nous permettent pas de déterminer si l'augmentation progressive de la prévalence de la multimorbidité avec l'âge relève de l'association de facteurs de risque généraux (en particulier d'un niveau de stress élevé ou du tabagisme) ou du contexte social (notamment du chômage et de l'inégalité entre les genres). Cette question mérite un examen plus poussé, en particulier parce que le chômage, les inégalités de revenu, le niveau d'activité physique et le stress sont des facteurs de risque évitables contre lesquels des actions multisectorielles pourraient être entreprises.

En troisième lieu, nous avons remarqué certaines tendances dans la prévalence des diverses affections chroniques. L'arthrite, les troubles de l'humeur ou l'anxiété et l'asthme étaient globalement les plus fréquentes, présentes dans toutes les combinaisons d'affections et toutes significativement plus fortement prévalentes chez les femmes. L'anxiété ou les troubles de l'humeur étaient présents pour plus de la moitié de la multimorbidité chez Canadiens les plus jeunes (20 à 34 ans), avec par ailleurs une fréquence des troubles mentaux plus élevée chez les jeunes femmes que chez les jeunes hommes.

En dernier lieu, nos résultats selon lesquels l'arthrite et les troubles mentaux constituent la paire la plus fréquente d'affections concomitantes vont dans le même sens que ceux d'une étude canadienne menée en 2012 qui a constaté que l'arthrite était l'une des principales affections en cas de bimorbidité ${ }^{24}$. En outre, notre analyse des paires et des triades d'affections les plus fréquentes met en évidence la nécessité d'examiner de manière plus approfondie les diverses grappes d'affections pour permettre aux programmes axés sur les soins globaux et intégrés d'être plus efficaces ${ }^{25}$.

\section{Forces et limites}

Même si la prévalence de la multimorbidité et les déterminants associés présentés ici sont valables pour l'ensemble de la population canadienne ne vivant pas en établissement, notre étude comporte certaines limites.

Nos résultats sont probablement sujets à un biais de mémoire, car les données 
sont basées sur l'autodéclaration de diagnostics cliniques. De plus, ils sont en partie dépendants de notre définition de cas opérationnelle de la multimorbidité, c'est-à-dire du nombre et du type d'affections chroniques prises en considération. Fortin et ses collaborateurs ${ }^{10}$ ont fait l'hypothèse que plus les affections retenues sont nombreuses, plus les estimations de la prévalence sont élevées. Il est vrai que Rapoport et ses collaborateurs $^{26}$ ont étudié 22 affections et ont fait état d'une prévalence de la multimorbidité au Canada plus élevée que Cazale et ses collaborateurs ${ }^{27}$, qui n'ont étudié que 7 affections. Fortin et ses collaborateurs ${ }^{10}$ ont cependant noté un effet de seuil : lorsque 15 affections ou plus sont prises en considération, cela n'a plus d'effet sur la prévalence. Pour notre part, nous avons retenu, en nous appuyant sur un ensemble de critères de santé publique, 9 affections chroniques parmi les affections relevées par l'ESCC. En raison de la nature exploratoire de nos analyses et des multiples comparaisons, une erreur de type 1 est probable.

Malgré ces limites, nos résultats contribuent à nourrir l'épidémiologie de la multimorbidité, ce qui contribue à mieux guider les efforts de prévention, à réduire le fardeau des affections chroniques et à harmoniser les services de soins de santé et les besoins globaux des patients. Les approches traditionnelles centrées sur une seule affection se révèlent de moins en moins valables, étant donné que la multimorbidité devient la norme chez les personnes atteintes d'affections chroniques. Une approche intégrée visant ces multiples affections et leurs facteurs en amont, notamment les déterminants socioéconomiques et les facteurs de risque comportementaux, est au contraire susceptible d'avoir un effet systématique de grande portée sur de nombreux enjeux, notamment la qualité de vie, les coûts associés aux soins de santé et la mortalité $^{16,28}$. D’autres études visant à faire la lumière sur les répercussions sociales et économiques de la multimorbidité sont nécessaires, même si nous pouvons d'ores et déjà supposer que tout constat dans ce domaine révélera des besoins en matière de prévention, particulièrement en ce qui concerne la lutte contre les facteurs de risque et les déterminants généraux des affections chroniques les plus fréquentes.

\section{Références}

1. Ronksley PE, Sanmartin C, Campbell DJ. Obstacles perçus à l'obtention de soins primaires chez les Canadiens de l'Ouest atteints de maladies chroniques. Rapports sur la santé. 2014;25:3-11.

2. Ford ES, Croft JB, Posner SF, Goodman RA, Giles WH. Co-occurrence of leading lifestyle-related chronic conditions among adults in the United States 2002-2009. Prev Chronic Dis. 2013;10:E60.

3. Organisation mondiale de la santé. Prévention des maladies chroniques: un investissement vital. Genève $(\mathrm{CH})$ : Organisation mondiale de la santé; 2005.

4. Starfield B. Challenges to primary care from co- and multi-morbidity. Prim Health Care Res Dev. 2011;12(1):1-2.

5. Organisation mondiale de la santé. Health statistics and information systems: projections of mortality and causes of death, 2015 and 2030 [Internet]. Genève $(\mathrm{CH})$ : Organisation mondiale de la santé; 2015 (consultation le 16 janvier 2015). Consultable en ligne à partir de la page; http://www.who. int/healthinfo/global_burden_disease/projections/en/

6. Statistique Canada. Les taux de mortalité normalisés selon l'âge, pour certaines causes de décès et le sexe (Les deux sexes) [Internet]. Ottawa (Ont.) : Statique Canada; [modification le 28 janvier 2014; consultation le $1^{\text {er }}$ décembre 2014]. Consultable à la page : http://www.statcan.gc.ca/tables-tableaux/ sum-som/102/cst01/health30a-fra.htm

7. Boyd CM, Fortin M. Future of multimorbidity research: how should understanding of multimorbidity inform health system design? Public Health Rev. 2010; 32(2):451-474.

8. Menotti A, Mulder I, Nissinen A, Giampaoli S, Feskens EJ, Kromhout D. Prevalence of morbidity and multimorbidity in elderly male populations and their impact on 10-year all-cause mortality: the FINE study (Finland, Italy, Netherlands, Elderly). J Clin Epidemiol. 2001;54(7):680-686.
9. Fortin M, Bravo G, G, Hudon C, Vanasse A, Lapointe L. Prevalence of multimorbidity among adults seen in family practice. Ann Fam Med. 2005;3 (3):223-228.

10. Fortin M, Stewart M, Poitras ME, Almirall MD, Maddocks H. A systematic review of prevalence studies on multimorbidity: toward a more uniform methodology. Ann Fam Med. 2012;10(2):142-151.

11. Agborsangaya $\mathrm{CB}$, Ngwakongnwi E, Lahtinen M, Cooke T, Johnson JA. Multimorbidity prevalence in the general population: the role of obesity in chronic disease clustering. BMC Public Health. 2013;13:1161.

12. Akner G. Analysis of multimorbidity in individual elderly nursing home residents. Development of a multimorbidity matrix. Arch Gerontol Geriatr.2009;49(3):413-419.

13. Marengoni A, Rizzuto D, Wang HX, Winblad B, Fratiglioni L. Patterns of chronic multimorbidity in the elderly population. J Am Geriatr Soc. 2009;57(2):225-230.

14. Schafer I, von Leitner EC, Schön G, et collab. Multimorbidity patterns in the elderly: a new approach of disease clustering identifies complex interrelations between chronic conditions. PLoS One. 2010;5(12):e15941.

15. Violán C, Foguet-Boreu Q, Roso-Llorach A, et collab. Burden of multimorbidity, socioeconomic status and use of health services across stages of life in urban areas: a cross-sectional study. BMC Public Health. 2014;14:530.

16. Salisbury C, Johnson L, Purdy S, Valderas JM, Montgomery AA. Epidemiology and impact of multimorbidity in primary care: a retrospective cohort study. Br J Gen Pract. 2011;61:e12-e21.

17. Béland Y. Enquête sur la santé dans les collectivités canadiennes : aperçu de la méthodologie. Rapports sur la santé. 2002;13(3):9-15.

18. Statistique Canada. Guide de l'utilisateur des données de l'Enquête canadienne sur les mesures de la santé (ECMS) : cycle 2 [Internet]. Ottawa (Ont.) : Statistique Canada; 2012 [consultation le 8 mai 2015]. Consultable à la page : http://www23.statcan.gc.ca/imdb-bmdi/ document/5071_D2_T1_V2-fra.htm

19. Betancourt MT, Roberts KC, Bennett TL, Driscoll ER, Jayaraman G, Pelletier L. Surveillance des maladies chroniques au Canada : Cadre conceptuel d'indicateurs des maladies chroniques. Maladies chroniques et blessures au Canada. 2014;34 suppl. 1:1-33. 
20. Agence de la santé publique du Canada. Groupe de travail sur la multimorbidité. Réunion technique: Mesure de la multimorbidité pour la surveillance des maladies chroniques au Canada - Rapport sommaire. Division de la surveillance et du contrôle des maladies chroniques, Centre de prévention des maladies chroniques, Agence de la santé publique du Canada. 2012 [Rapport non publié].

21. Ward BW, Schiller JS. Prevalence of multiple chronic conditions among US adults: estimates from the National Health Interview Survey, 2010. Prev Chronic Dis. 2013;10:E65.

22. Parekh AK, Goodman RA, Gordon C, Koh HK. Managing multiple chronic conditions: a strategic framework for improving health outcomes and quality of life. Public Health Rep. 2011;126(4):460-471.

23. Barnett K, Mercer SW, Norbury M, Watt G, Wyke S, Guthrie B. Epidemiology of multimorbidity and implications for health care, research, and medical education: a crosssectional study. Lancet. 2012;380(9836):37-43.

24. Agborsangaya $C B$, Lau $D$, Lahtinen $M$, Cooke T, Johnson JA. Multimorbidity prevalence and patterns across socioeconomic determinants: a cross-sectional survey. BMC Public Health. 2012;12:201.

25. Kronick RG, Bella M, Gilmer TP, Somers SA. The faces of Medicaid II: recognizing the care needs of people with multiple chronic conditions. Hamilton (NJ) : Center for Health Care Strategies; 2007.

26. Rapoport J, Jacobs P, Bell NR, Klarenbach S. Pour une mesure plus précise du fardeau économique associé aux maladies chroniques au Canada. Maladies chroniques au Canada. 2004;25(1):15-23.

27. Cazale L, Dumitru V. Les maladies chroniques au Québec : quelques faits marquants. Zoom Santé. 2008;29:1-4.

28. Goodman RA, Posner SF, Huang ES, Parekh AK, Koh HK. Defining and measuring chronic conditions: imperatives for research, policy, program and practice. Prev Chronic Dis. 2013;10:E66. 